\title{
STUDIES ON EFFECTS OF PYRAMID STIMULATION UPON FLEXOR AND EXTENSOR MOTONEURONES AND GAMMA MOTONEURONES
}

\author{
Masamichi Kato, Haruo TAKAMURA \\ AND Bun'ichi FuJIMORI* \\ Department of Physiology, Hokkaido University, \\ School of Medicine, Sopporo, Japan
}

Numerous papers have been published on the effects of pyramidal tract stimulation upon spinal motoneurones. However, it must be noticed in such an experiment that extrapyramidal effects may inevitably intermingle with the pyramidal effects in the case of motor cortex stimulation, unless all the bulbar structures except the bulbar pyramid have been completely destroyed.

Even in the case of bulbar pyramid stimulation, the following three points must be taken into consideration as possible causes of indirect effects of the stimulation upon motoneurones: (1) Adjacent bulbar structures especially the medial lemniscus may be stimulated due to current spread. (2) Impulses of pyramidal fibers elicited by the stimulation may also reach extrapyramidal structures in the brain stem through their collaterals. (3) Spinal motoneurones may be stimulated with impulses through the gamma-loop at the spinal segmental level. Few works seem to have been done under strict experimental conditions to eliminate all these possible indirect effects.

In the present experiment, therefore, an attempt has been made to analyse the effect of pyramid stimulation upon extensor and flexor motoneurones and gamma motoneurones innervating the hindlimb of cats under such strict experimental conditions.

\section{METHODS}

Experiments were carried out on 40 cats $(2.7-4.5 \mathrm{~kg})$.

The spinal cord was exposed in the usual manner under ether anesthesia. Cats were mounted in a stereotaxic instrument of 'Noken' type. In order to eliminate all the possible indirect effects noted above, all the structures of the medulla and pons except the pyramidal tract were entirely removed including the cerebellum by means. of electrocoagulation and suction. The pyramidal tract was transected at the midpontine level. All the ventral roots from L4 downwards were cut bilaterally in most cases and unilaterally in the remaining cases.

Received for publication September 28, 1963.

* 加藤正道, 高村春雄, 藤森聞一 
Artificial respiration was started soon after the respiration ceased during the operation. The exposed lumbosacral spinal cord was bathed in a warmed mineral oil, the temperature of the oil pool being maintained at $36-37^{\circ} \mathrm{C}$ by an infra-red lamp. Records were taken at least two hours after cessation of ether anesthesia.

As to the method of pyramid stimulation, coaxial needle electrode of steel tube of $500 \mu$ in diameter containing in it a needle of $100 \mu$ in diameter insulated except at the tip was employed. The electrode was introduced into the pyramidal tract by means of a micromanupulator; the vertical as well as longitudinal steps of exploration were spaced by $1 \mathrm{~mm}$, while laterally the areas in $0.5,1.0,2.0$ and $0.3 \mathrm{~mm}$ distance from the mid-line were explored on both sides.

Square wave pulse stimulation of $0.5 \mathrm{msec}$ duration was delivered to the pyramid, the intensity (less than $3.0 \mathrm{v}$ ) and frequency $(1-500 / \mathrm{sec})$ being varied.

Mono- and polysynaptic reflexes of both extensor and flexor motoneurones were picked up from the L7 or S1 ventral root, square wave pulse of submaximal intensity of $0.5 \mathrm{msec}$ duration and $0.5 / \mathrm{sec}$ frequency being applied to either the tibial or peroneal nerve at the popliteal fossa. Firing index by Lloyd and MCINTyre (1955) and spontaneous discharges of motoneurones were obtained from L7 or S1 ventral root thin filaments. Firing index was calculated from 30 trials at each points, discharge rate being kept at approximately $50 \%$ in the control record.
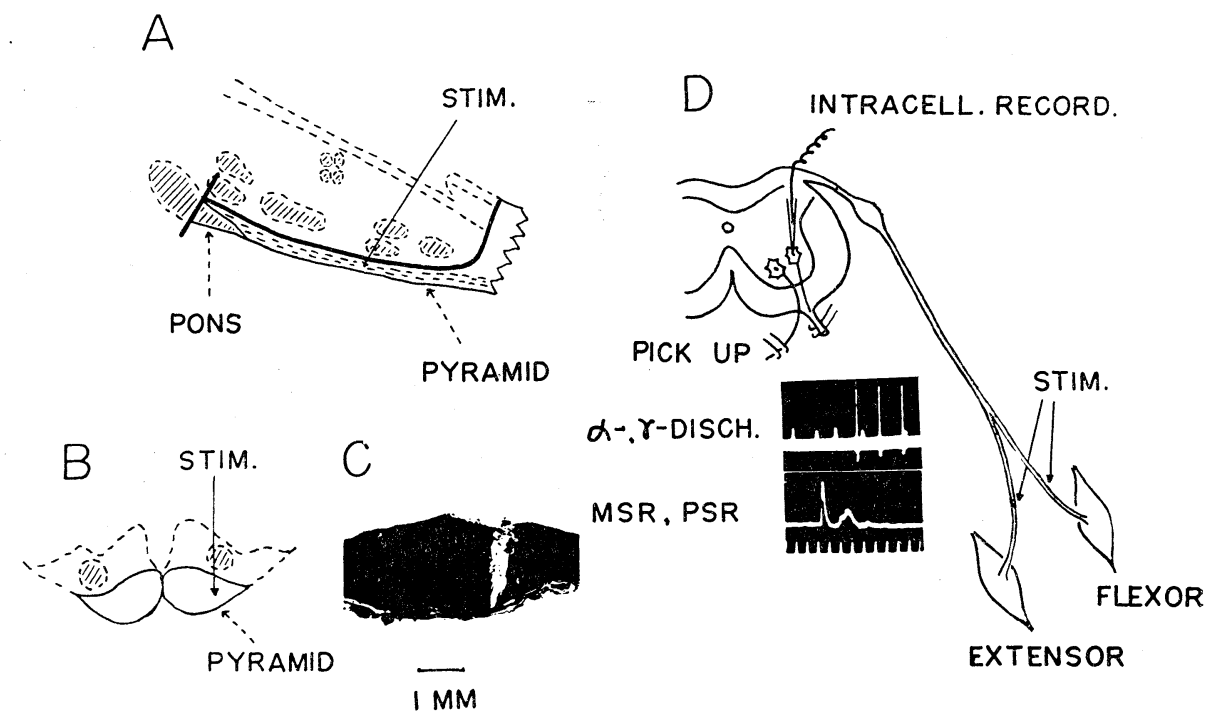

FIG. 1. Schema showing the experimental methods.

A : Medullary and pontine structures except the pyramidal tract were entirely removed by means of electrocoagulation and suction, and the pyramidal tract was transected at the midpontine level. B: Frontal plane of the pyramid showing effective stimulating points. C: Histological view of the stimulating points in the pyramid. D: Monosynaptic and polysynaptic reflex discharges from both extensor and flexor motoneurones were picked up from L7 or S1 ventral root with stimulation either of the peroneal or tibial nerve at the popliteal fossa. Unit $\alpha$ - and $\gamma$-discharges were also picked up from L7 or S1 ventral root filaments. Intracellular recording of individual motoneurones was made by the use of glass capillary microelectrodes. 
Intracellular microelectrode technique was also employed to analyse the effect of pyramid stimulation upon individual motoneurones. For this purpose, glass capillary micropipetts filled with $3 \mathrm{M} \mathrm{KCl}$ and having 5-20 $\mathrm{M} \Omega$ resistance were used. In this series of experiments, cats were immobilized with Flaxedil.

Discharges of gamma motoneurones were picked up from ventral root thin filaments, the identification of which was made by the conduction velocity and their amplitudes. The experimental set-up is shown in FIG. 1.

\section{RESULTS}

(1) Effective points of stimulation in the pyramid. As the first step of the experiments, exploration was made to find out the effective points of stimulation upon mono- and polysynaptic reflexes of flexor and extensor motoneurones with repetitive stimulation of $50-100 / \mathrm{sec}$ frequency.

It was found that the most effective points were distributed in the longitudinal plane $1.0 \mathrm{~mm}$ lateral from the midline and $6.0-7.5 \mathrm{~mm}$ depth from the bottom of the fourth ventricle. A case of histological observation is shown in FIG. 1C.

(2) Effects of repetitive stimulation of the pyramid upon mono- and pyly-synaptic reflexes of flexor and extensor motoneurones. In a series of experiments, repetitive stimulation of varied frequencies were delivered to the pyramid, intensity being kept constant.

With increase in frequency of stimulation, increase in amplitude of the monosynaptic reflex discharge from flexor motoneurones up to $150-200 \%$ was observed in all 16 tested cases, while slight decrease in amplitude of monosynaptic reflex discharge from extensor motoneurones was recognized in 12 out of 16 tested cases, one example being shown in FIG. 2. Maximum changes were obtained at $100-200 / \mathrm{sec}$ frequencies in either case.

In another series of experiments on 6 cats, repetitive stimulation of varied intensities was applied likewise, frequency being kept constant. With increase in intensity of stimulation, the flexor monosynaptic reflex discharge increased in amplitude while the extensor one decreased. No reversal of the effect was observed in either case.

Polysynaptic reflexes of flexor and extensor motoneurones showed almost the same directions of changes respectively as the above noted changes in monosynaptic reflex responses of extensor and flexor motoneurones.

To summarize, the effects of pyramid stimulation upon mono- and polysynaptic reflexes of flexor and extensor motoneurones were reciprocal: facilitatory effects being observed in the case of flexor motoneurones while inhibitory ones in the case of extensor. 


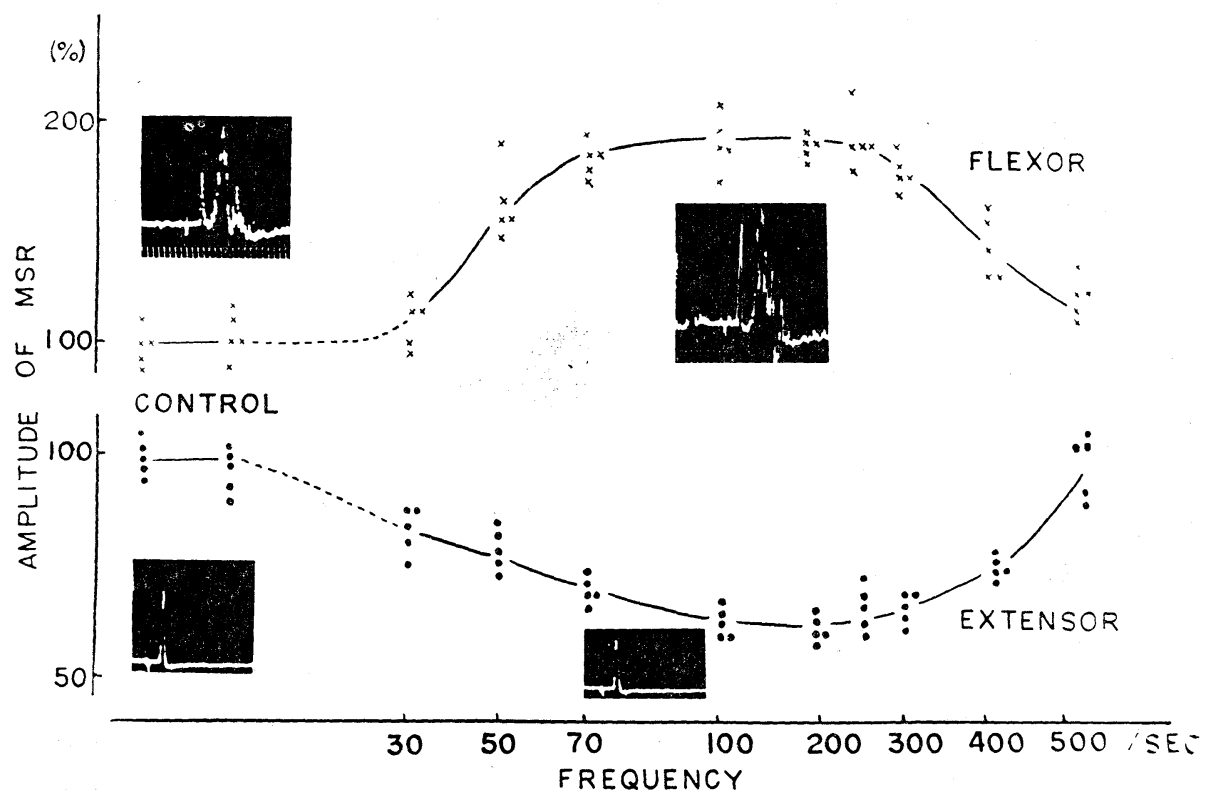

FIG. 2. Effects of pyramid stimulation with varied frequencies upon amplitudes of monosynaptic reflex discharges from flexor and extensor motoneurones.

Abscissa: frequency of pyramid stimulation which was applied to the pyramid for 10 second with constant intensity. Ordinate: percentage of amplitude of monosynaptic reflex discharge. Monosynaptic reflex discharges from flexor motoneurones increase in ampiitude, whereas those from extensor ones decrease in amplitude with increase in frequency of stimulation, showing their maximum changes at 100-200/ sec frequencies in both cases.

(3) Effects of single shock stimulation of the pyramid upon monosynaptic reflexes of flexor and extensor motoneurones. In order to analyse further the effects of pyramid stimulation upon flexor and extensor motoneurones, interaction between pyramidal and peripheral nerve stimulations was investigated, conditioning shock and test shock being applied respectively to the pyramid and the peroneal or tibial nerve at varied intervals.

In the case of flexor monosynaptic reflex response, initial slight fluctuation at about $6 \mathrm{msec}$ was followed by a facilitatory effect for $30-40 \mathrm{msec}$ in all 10 tested cases. Whereas in the case of extensor motoneurones, monophasic inhibitory effect for about $30 \mathrm{msec}$ and inhibitory effect for about $30 \mathrm{msec}$ with initial slight facilitatory one were recognized respectively in 14 and 3 of 17 tested cases. FIG. 3 shows a case of the result.

As a whole, reciprocal effect was also observed in this series of experiment, that is to say, facilitatory effect upon flexor motoneurones and inhibitory effect upon extensor ones were recognized as in the above noted case with repetitive stimulation. 


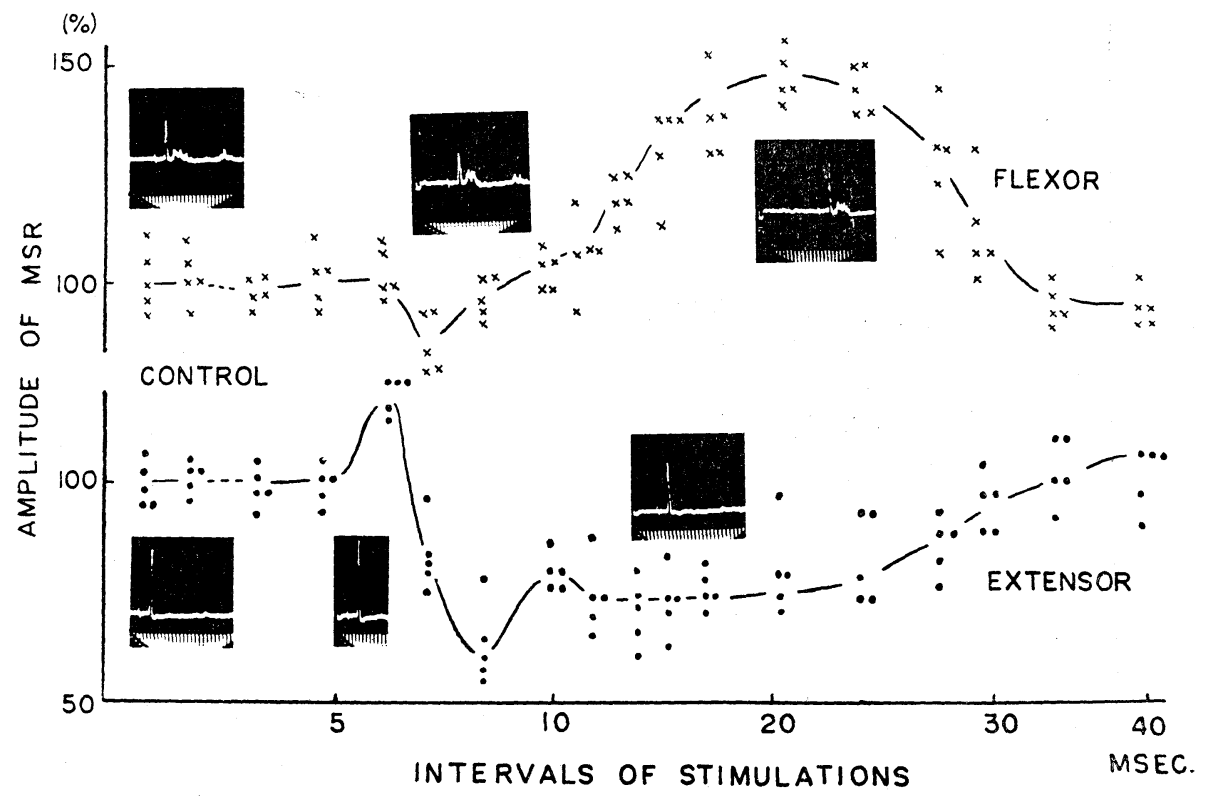

FIG. 3. Interrelationship between effects of pyramid and peripheral nerve stimulations with varied intervals upon monosynaptic reflex discharge from flexor and extensor motoneurones.

Abscissa: intervals between conditioning shocks to the pyramid and test shocks to the peroneal or tibial nerve. Ordinate: percentages of amplitude of monosynaptic reflex discharges. A facilitatory effect is observed for about 30 msec in the flexor monosynaptic reflex response with slight initial fluctuation starting from an interval of $6 \mathrm{msec}$. The extensor monosynaptic reflex response shows initial slight facilitation followed by distinct inhibition for approximately $30 \mathrm{msec}$.

(4) Effects of single pulse stimulation of the pyramid upon individual motoneurones. In order to analyse the effects of pyramid stimulation upon individual motoneurones, firing index was measured on 13 unit flexor and unit extensor motoneurones. In flexor motoneurones, a facilitatory effect was observed with a latency of $6 \mathrm{msec}$ in all cases, whereas in extensor motoneurones an inhibitory effect for about $30 \mathrm{msec}$ with initial facilitatory effect at about $6 \mathrm{msec}$ was observed in all cases. An example is shown in Fig. 4.

Intracellular recordings were made of 12 flexor and 16 extensor motoneurones. EPSPs were evoked in 12 flexor and 6 extensor motoneurones with latencies o $5.0-10.0 \mathrm{msec}$, on the other hand IPSPs were obtained in 8 extensor motoneurones with latencies of 7.5-11.0 msec and mixed form of EPSP and IPSP were observed in 2 extensor motoneurones with latencies of 6.0-7.0 msec. Examples are shown in FIG. 5. 


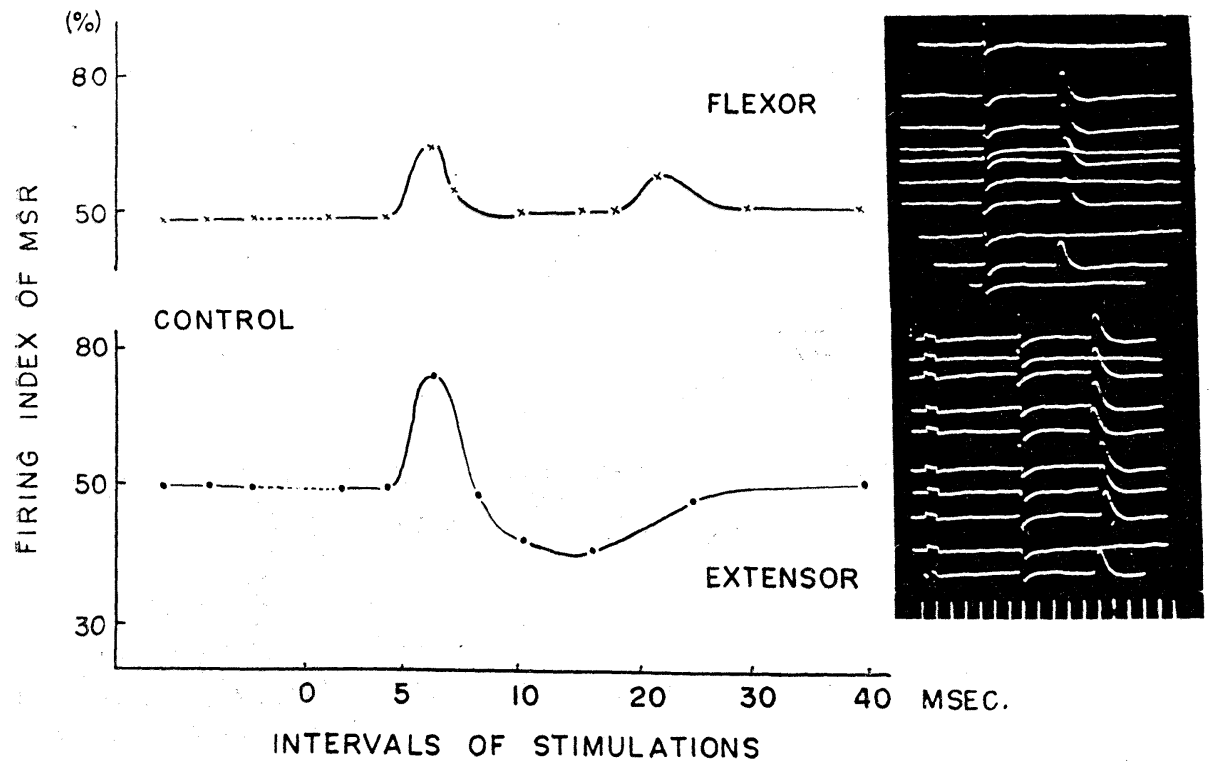

FIG. 4. Effects of single pyramid stimulation upon firing indices of extensor and flexor motoneurons.

Abscissa: intervals between conditioning stimulations of the pyramid and test shocks of the peripheral nerves. Ordinate: firing indices. In the case of flexor motoneurones, initial facitatory effect is observed at $6 \mathrm{msec}$. Whereas in the case of extensor motoneurones, initial facilitatory effect is followed by inhibitory one for approximately $30 \mathrm{msec}$.

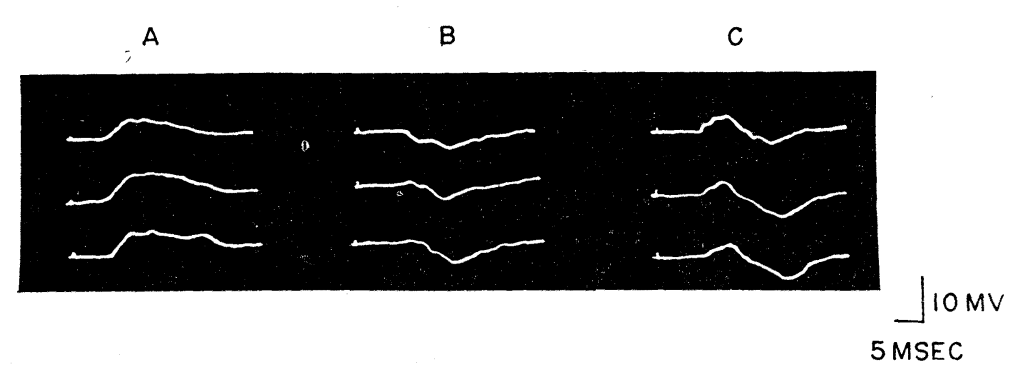

FIG. 5. Intracellular recordings from flexor and extensor motoneurones.

Each column shows three successive recordings. In A (flexor, resting membrane potential: $70 \mathrm{mV}$ ), EPSPs appear at latency of $6 \mathrm{msec}$, in $\mathrm{B}$ (extensor, resting membrane potential: $53 \mathrm{mV}$ ) IPSPs at $8 \mathrm{msec}$ and in $\mathrm{C}$ (extensor, resting membrane potential : $63 \mathrm{mV}$ ) mixed form of EPSP and IPSP at $6 \mathrm{msec}$.

(5) Effects of pyramid stimulation upon motoneurone discharges. Single or repetitive stimulation was applied to the pyramid to observe the discharge patterns of motoneurones produced by the stimulation or the effects upon their spontaneous discharges. As a result, no discharge of motoneurone was elicited 
with single pulse stimulation even at the intensity as high as 30 volts, while with repetitive stimulation higher than $30 / \mathrm{sec}$ frequency initiation of discharges was observed in all the 30 tested cases at low intensities of stimulation around 3 volts.

Facilitatory effect upon spontaneous discharges of motoneurones (increase in frequency of discharge) was observed in 3 tested flexor motoneurones, whereas inhibitory one (decrease in frequency) was recognized in 2 out of 4 extensor motoneurones, the remaining 2 cases showed no effect. FIG. 6 indicates a case of the experimental result.

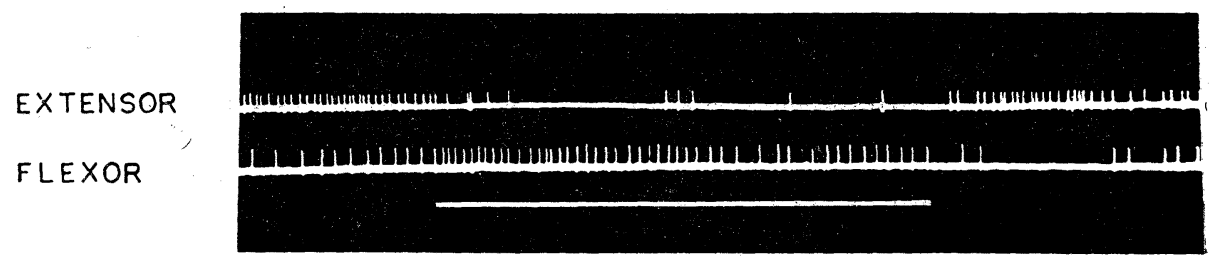

FIG. 6. Effects of pyramid stimulation upon tonic $\alpha$-discharges of both extensor and flexor motoneurones.

During the pyramid stimulation the frequency of discharges of the extensor motoneurone decreases, while the flexor one increases.

(6) Effects of pyramid stimulation upon gamma discharges. Effects of repetitive stimulation of the pyramid upon gamma discharges were examined in 30 gamma neurones, frequency $(30-150 / \mathrm{sec})$ and intensity $(0-3.0 \mathrm{v})$ of the stimulation being varied.
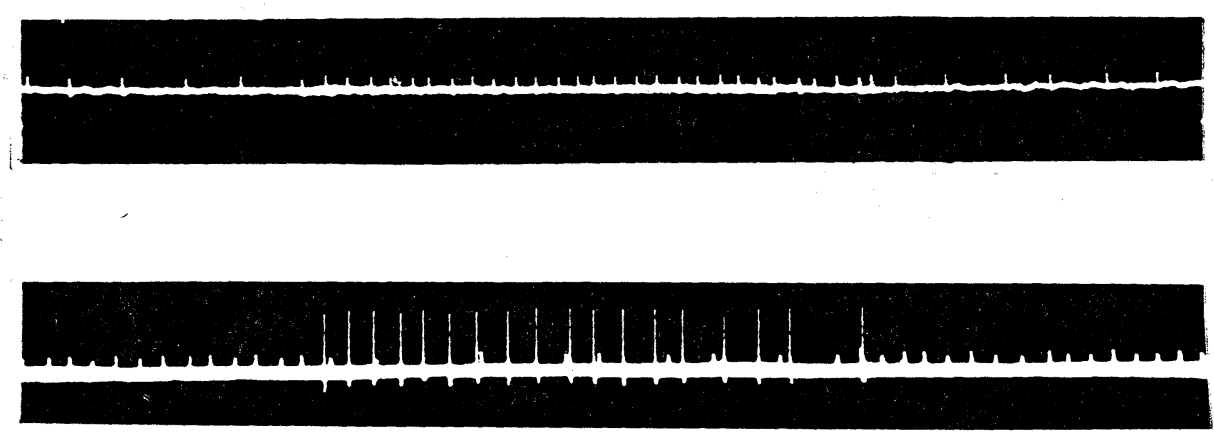

FIG. 7. Effects of pyramid stimulation upon $\gamma$-motoneurones.

Two cases of effects are shown in this figure. Upper trace: $\gamma$-discharges increase in frequency during the stimulation (50/sec frequency, $1 \mathrm{msec}$ pulse duration and $2.0 \mathrm{~V}$ intensity) for 1 second. Lower trace: $\gamma$-discharges decrease in frequency in association with initiation of $\alpha$-discharges, larger spikes, during the stimulation indicated by a black bar. 


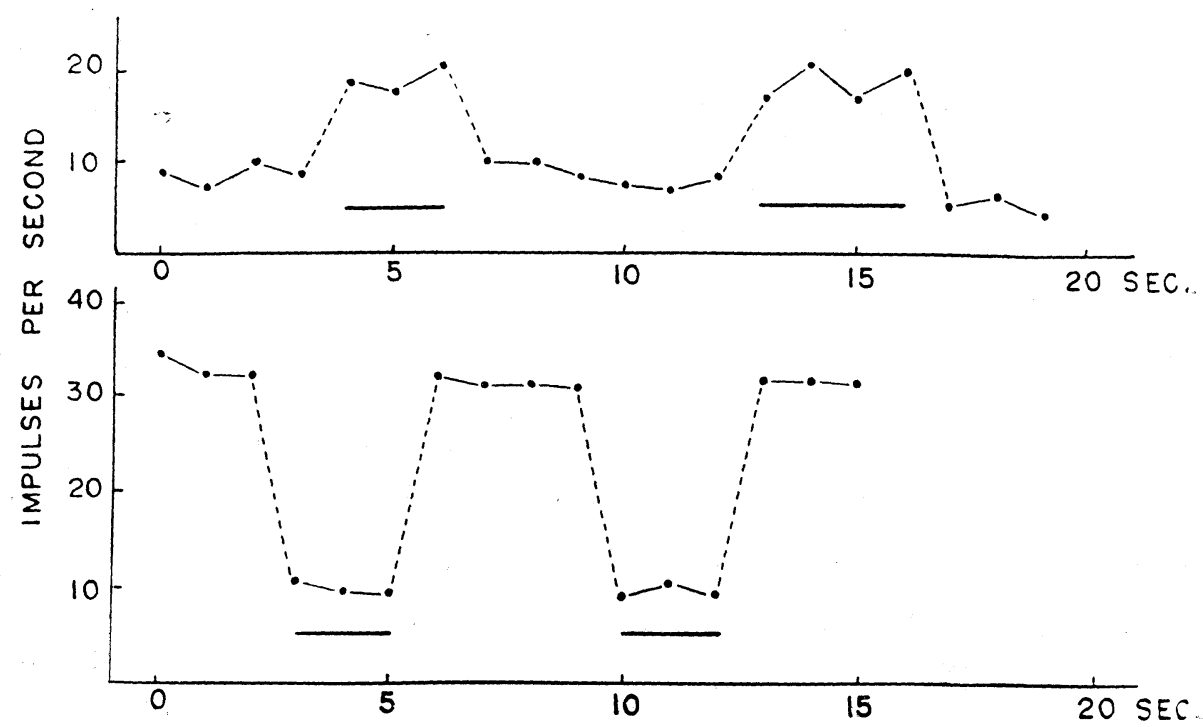

FIG. 8. A graphic representation of the effect of the pyramid stimulation upon the discharge frequency of unit $\gamma$-motoneurone.

Abscissa: time in second. Ordinate: discharge frequency in one second. Black bars show the pyramid stimulation ( $50 / \mathrm{sec}$ frequency, $1 \mathrm{msec}$ pulse duration and 2.0 volt intensity). In the upper graph, $r$-discharge increases during the stimulation, and in the lower graph it decreases during the stimulation.

Inhibitory effects (decrease in frequency of discharge) were observed in 10 gamma neurones and facilitatory one in 4 neurones by stimulation of the contralateral pyramid for $1-10 \mathrm{sec}$. Remaining 16 neurones which were spontaneously discharging showed no response to the stimulation.

Throughout the experiments, it was observed that the effect became more marked with increase in intensity and frequency of stimulation, irrespective of the direction of the effects. Furthermore, neither change in the direction of the effects nor after-effect was observed with varied parameters of the stimulation. It was also noticed that no strict localization of effective points in the pyramid was observed in this series of experiment as in the case of alpha motoneurones. FIGs. 7 and 8 illustrate a case of this series of experimental results.

\section{DISCUSSION}

(1) In the present experiments, effective points of stimulation upon hindlimb motoneurones were found to be situated in a restricted region of the pyramid. This result may provide an evidence that pyramidal tract fibers are divided, to a certain extent, into segmental groups at the medullary level.

(2) As to the effect of pyramidal tract stimulation upon spinal moto- 
neurones, SASAKI, NAMIKAWA and MATSUNAGA (1960) reported that solely EPSP and spike potential were obtained with stimulation of the pyramidal decussation in cats. On the other hand, PREsTon and Whitlock $(1960,1961)$ observed generalized facilitatory or inhibitory effects upon flexor and extensor monosynaptic reflexes with stimulation of the precentral gyrus in monkey and EPSP as well as IPSP were obtained from individual spinal motoneurones.

Recently LUNDBERG and VOORHOEve (1962) carried out exhaustive experiments in cats along this line and found that facilitatory effect was more marked on the flexor monosynaptic reflex than on the extensor one with stimulation of sensorimotor cortex, and that the ratios of EPSP and IPSP were $10: 3$ in the case of flexor motoneurones, whereas $4: 13$ in the case of extensor ones.

In the present experiment which was carried out under strict experimental conditions to observe the direct effect of pyramid stimulation upon spinal motoneurones, (1) facilitatory effect was observed from the statistical view point in all the tested cases of flexor motoneurones, whereas inhibitory one was frequently observed in the case of extensor motoneurones judging from the effects of the stimulation upon their monosynaptic reflexes. (2) as to the effect upon individual motoneurones analyzed by means of the firing index method as well as intracellular microelectrode technique, facilitatory effect was obtained in all the tested cases of flexor motoneurones, whereas inhibitory one was frequently observed in the case of extensor motoneurones. These results agree essentially with those of LUNDBERG and VOORHOEvE (1962) which were obtained with stimulation of the cerebral cortex.

Some discrepancies between their results and ours may be attributable to certain differences in the experimental conditions.

At any rate, on the basis of the results obtained in the present experiment, it may be said that the pyramidal tract as a whole has more powerful control over flexor motoneurones as postulated by LUNDBERG and VOORHOEVE, and that extensor motoneurones may be under the influence of the reciprocal inhibition from the above noted facilitatory pyramidal fibers to flexor motoneurones.

(3) As to the effects upon gamma motoneurones, ElDRED, GRANIT and MERTON (1953) reported that the inhibitory effects were observed by stimulation of contralateral internal capsule. However, in such an experiment, extrapyramidal effect upon gamma motoneurones can not be excluded, as postulated by APPELBERG (1962) who obtained inhibitory effect upon gamma motor system following stimulation of the red nucleus.

In the present experiment which was carried out under the conditions of eliminating all the possible indirect effects through the extrapyramidal system, facilitatory as well as inhibitory effects were observed in half of the tested cases. This result suggests that the pyramidal tract actually includes fibers 
which belong to the gamma system.

\section{SUMMARY}

In order to analyse the function of the pyramidal tract, studies were made on the effects of pyramid stimulation upon flexor and extensor motoneurones and also upon gamma motoneurones innervating the hindlimb of cats.

Special care was taken to eliminate all the possible indirect effects of pyramid stimulation upon the above noted neurones due to current spread to the structure in the medulla, through collaterals of pyramidal fibers to the structure of the brain stem and also through the gamma-loop at the spinal segmental level. For this purpose, medullar and pontine structures except the pyramidal tract was entirely removed by means of electrocoagulation and suction, and the pyramidal tract was transected at the midpontine level. All ventral roots from L4 downwards were cut bilaterally or unilaterally.

1. Effective points of stimulation upon spinal motoneurones were found to be distributed in the contralateral, vertical plane in the pyramid $1.0 \mathrm{~mm}$ lateral from the midline. However, those upon gamma motoneurones were dispersed rather diffusely in the pyramid.

2. With repetitive stimulation of the pyramid monosynaptic and polysynaptic reflex discharges from flexor motoneurones increased in amplitude in all 16 tested cases, whereas those from extensor ones showed appreciable decrease in amplitude in 12 out of 16 tested cases.

3. Following single pulse conditioning stimulation of the pyramid, increase in amplitude of the flexor monosynaptic reflex discharge was observed for 30-40 msec in all 10 tested cases. Whereas, monophasic decrease in amplitude for about $30 \mathrm{msec}$ and inhibitory effect for about $30 \mathrm{msec}$ with initial slight facilitatory one were recognized respectively in 14 and 3 of 17 tested cases of extensor motoneurones.

4. As to the effect of pyramid stimulation upon individual motoneurones, facilitatory effect was obtained in the case of flexor motoneurones, whereas inhibitory one was observed frequently in the case of extensor ones judging from the firing index method.

On the other hand, EPSPs were evoked in 12 flexor and 6 extensor motoneurones, IPSPs were evoked in 8 extensor ones and mixed form of EPSP and IPSP were observed in 2 extensor motoneurones.

5. These results provide basis for the suggestion that the pyramidal tract as a whole has a stronger facilitatory control over flexor motoneurones than over extensor ones and that these facilitatory pyramidal fibers to flexor motoneurones may exert a certain inhibitory effect upon extensor motoneurones.

6. Facilitatory effect upon gamma neurones: increase in frequency of spontaneous discharges or initiation of discharges, as well as inhibitory effect: 
decrease in frequency of spontaneous discharges, were observed respectively in 4 and 10 out of 30 tested cases with pyramid stimulation.

The present experiment was supported by grants from the Rockefeller Foundation and the Japanese Ministry of Education.

\section{REFERENCES}

1) Appelberg, B. (1962). The effect of electrical stimulation of nucleus ruber on the gamma motor system. Acta physiol. scand., 55: 150-159.

2) Eldred, E., Granit, R. and Merton, P. A. (1953). Supraspinal control of the muscle spindle and its significance. J. Physiol., 122: 498-523.

3) Lloyd, D. P.C. And McIntiRe, A.K. (1955). Monosynaptic reflexes of individual motoneurones. J. gen. Physiol., 38: 771-787.

4) Lundberg, A. And Voorhoeve, P. (1962). Effects from the pyramidal tract on spinal reflex arcs. Acta physiol. scand., $56: 201-219$.

5) Preston, J.B. And Whitlock, D. G. (1960). Precentral facilitation and inhibition of spinal motoneurones. J. Neurophysiol., $23: 154-170$.

6) Preston, J.B. And Whitlock, D. G. (1961). Intracellular potentials recorded from motoneurones following precentral gyrus stimulation in primates. J. Neurophysiol., $24: 91-100$.

7) Sasaki, K., Namikawa, A. And Matsunaga, M. (1960). Effects of stimulation of the pyramidal tract and striate body upon spinal motoneurones. Jap. J. Physiol.,. $10: 403-413$. 